\title{
ORIGINAL ARTICLE \\ Sleep disruption in tetraplegia: a randomised, double-blind, placebo-controlled crossover trial of $\mathbf{3} \mathbf{~ m g}$ melatonin
}

\author{
J Spong ${ }^{1}$, GA Kennedy ${ }^{1,2,3}$, J Tseng ${ }^{4}$, DJ Brown ${ }^{5}$, S Armstrong ${ }^{3,6}$ and DJ Berlowitz ${ }^{1,5,7}$
}

Study design: Randomised, double-blind, placebo-controlled crossover trial of melatonin supplementation to people with complete tetraplegia.

Objectives: To investigate the effect that $3 \mathrm{mg}$ melatonin supplementation has on objective and subjective sleep, quality of life and mood of people living with complete tetraplegia.

Setting: Austin Hospital Sleep Laboratory and participants' homes, Melbourne, Victoria, Australia.

Methods: Two week run-in followed by 3 week nightly administration of $3 \mathrm{mg}$ melatonin or placebo, 2-week washout and further 3 week administration of the opposite treatment. Four testing sessions were conducted; the last nights of the run-in, treatment and washout periods. Testing sessions involved recording full polysomnography, completing a questionnaire battery and collecting urine and blood samples. The questionnaires assessed mood, sleep symptoms and health-related quality of life, and the urine and plasma samples assayed 6-sulphatoxymelatonin (aMT6s) and melatonin levels, respectively. A sleep diary was completed throughout the study. Results: Eight participants (mean (s.d.): age 49.5 years (16), postinjury 16.9 years (7.1)) were recruited in which seven concluded the protocol. Endogenous-circulating melatonin was significantly higher $(P \leqslant 0.01)$ following melatonin (urine: $152.94 \mu \mathrm{h}^{-1}$ (74.51), plasma: $43554.57 \mathrm{pM}(33527.11)$ ) than placebo (urine: $0.86 \mu \mathrm{g} \mathrm{h}^{-1}$ (0.40), plasma: 152.06 pM (190.55)). Subjective sleep improved significantly following melatonin specifically for duration of sleep per night and psychological wellbeing. Objective sleep showed a significant increase in light sleep with melatonin, with all other sleep parameters being unchanged.

Conclusion: These results suggest that increasing melatonin in people with complete tetraplegia is beneficial, especially for subjective sleep. Investigation of the pharmacokinetics of melatonin metabolism in this population is warranted.

Sponsorship: This project is proudly supported by the Transport Accident Commission.

Spinal Cord (2014) 52, 629-634; doi:10.1038/sc.2014.84; published online 3 June 2014

\section{INTRODUCTION}

People living with a spinal cord injury report higher rates of sleep disturbances than the general population, ${ }^{1,2}$ which adversely affects their quality of life, especially in tetraplegia. ${ }^{3}$ The complex causes of sleep disruption in spinal cord injury have been recently reviewed, ${ }^{4}$ but one area of disruption that may be readily addressed is melatonin.

Melatonin, secreted by the pineal gland, has a major role in the timing of circadian rhythms, like the sleep-wake cycle. ${ }^{5}$ Melatonin secretion is influenced by the daily light-dark cycle, with light suppressing and darkness stimulating this process. In healthy adults, melatonin levels increase 2-3 h before sleep onset, peaking from 02:00 to $04: 00 \mathrm{~h}$. The average peak melatonin concentration at night is $60 \mathrm{pg} \mathrm{ml}^{-1}(260 \mathrm{pM})$, which gradually declines to $10 \mathrm{pg} \mathrm{ml}^{-1}(40 \mathrm{pM})$ during daytime. The daily melatonin rhythm is regulated by the suprachiasmatic nucleus ('circadian clock') which sends signals to the pineal gland via a circuitous route which involves other hypothalamic nuclei, brain stem nuclei, the spinal cord and peripheral sympathetic neurons from the superior cervical ganglion. The fibres to the superior cervical ganglion are routed along with those of the autonomic nervous system and it is this anatomical connection which is interrupted in complete tetraplegia. Subsequently, melatonin production following complete tetraplegia has been found to be undetectable. $^{6}$

Disrupted melatonin secretion following tetraplegia ${ }^{5}$ significantly reduces total sleep time and sleep efficiency, and increases rapid-eyemovement sleep latency, ${ }^{7}$ Timed, daily administration of melatonin improves sleep in able-bodied ${ }^{8}$ and functionally blind people ${ }^{9}$ and recently, an uncontrolled study of nightly melatonin supplementation in complete tetraplegia ${ }^{10}$ reported improved subjective sleep quality and an objective increase in rapid-eye-movement latency and stage 2 sleep. The present study sought to further explore the effect of exogenous melatonin supplementation on subjective and objective sleep for people with complete tetraplegia but using a randomised, blinded, placebo-controlled, crossover design.

\section{MATERIALS AND METHODS}

\section{Participants}

People with chronic ( $>1$ year) complete tetraplegia (American Spinal Injury Association Impairment Scale grade $\mathrm{A})^{11}$ between $18-70$ years of age were identified. Exclusion criteria included: pregnancy or breastfeeding, use of over-

\footnotetext{
${ }^{1}$ Institute for Breathing and Sleep, Austin Hospital, Melbourne, Victoria, Australia; ${ }^{2}$ Psychology Department, College of Arts, Victoria University, Melbourne, Victoria, Australia; ${ }^{3}$ The Bronowski Institute of Behavioural Neuroscience, Kyneton, Victoria, Australia; ${ }^{4}$ Department of Emergency Medicine, The Northern Hospital, Melbourne, Victoria, Australia; ${ }^{5}$ Spinal Research Institute, Austin Health, Melbourne, Victoria, Australia; ${ }^{6}$ Epworth Sleep Centre, Melbourne, Victoria, Australia and ${ }^{7}$ Department of Medicine, Austin Health and Northern Health, University of Melbourne, Melbourne, Victoria, Australia

Correspondence: Dr DJ Berlowitz, Institute for Breathing and Sleep, Bowen Centre, Austin Health, Heidelberg, Victoria 3084, Australia.

E-mail: david.berlowitz@austin.org.au
}

Received 3 October 2013; revised 3 April 2014; accepted 1 May 2014; published online 3 June 2014 
the-counter melatonin or travel across three or more time zones within 3 weeks of the study. Usual medications and medical sleep devices were continued during the trial. The Austin Health Human Research Ethics Committee approved the project and informed consent was obtained from all participants. The study is registered at www.anzctr.org.au (308365). We certify that all applicable institutional and governmental regulations concerning the ethical use of human volunteers were followed during the course of this research.

\section{Study design and procedure}

This study involved a 2-week run-in period, 3 weeks of $3 \mathrm{mg}$ per day melatonin or placebo (Compoundia, Melbourne Docklands, Melbourne, Australia), a 2week washout followed by the alternate treatment for another 3 weeks. Randomisation, blocking and balancing of treatment order was conducted externally by a clinical trials pharmacist. Capsules were ingested $2 \mathrm{~h}$ before usual sleep time. Four testing sessions were conducted $2 \mathrm{~h}$ before the participant's usual sleep time; on the last night of the run-in, washout and treatment periods, either in the participants' homes or the sleep laboratory according to participant preference. Testing was conducted under dim-light conditions. Participants were contacted weekly to screen for adverse events.

Two urine samples $(4 \mathrm{ml})$ were collected for home-based studies; one sample $2 \mathrm{~h}$ before usual sleep time and one upon awaking the following morning. For sleep laboratory studies, urine samples were collected hourly between 18:00 and 07:00 h. One blood (plasma) sample was collected $1 \mathrm{~h}$ before usual sleep time and immediately separated by centrifugation. Samples were stored at $-20^{\circ} \mathrm{C}$.

Urinary 6-sulphatoxymelatonin (aMT6s) was assayed by RIA ${ }^{12}$ using reagents obtained from Stockgrand (Guildford, Surrey, UK). The sensitivity of the assay was $2 \mathrm{ng} \mathrm{ml}^{-1}$ and the intra-assay and inter-assay coefficients of variation were $<15 \%$. Plasma melatonin $(200 \mu \mathrm{l})$ was assayed by RIA $^{13}$ using reagents obtained from Buhlmann Laboratories (Schönenbuch, Switzerland). The sensitivity of the assay was $8.6 \mathrm{pM}$ and intra-assay and the inter-assay coefficients of variation were $<15 \%$.

Objective sleep was assessed by recording overnight polysomnography (PSG), using the Compumedics Somte PSG (Compumedics Somte, Abbotsford, Australia). PSG parameters included central (C4/A1, C3/A2) electroencephalography, bilateral electro-oculography, electromyography (chin, diaphragmatic), electrocardiography, blood oxygen saturation, nasal pressure, leg movements, body position and respiratory movements (chest and abdomen). Sleep was staged, respiration scored and summary statistics generated by a sleep scientist according to international standard criteria. ${ }^{14,15} \mathrm{~A}$ rectal thermoresistor-measured core body temperature during the PSG. Room temperature was recorded before sleep.

Subjective sleep was assessed via responses on the Basic Nordic Sleepiness Questionnaire (BNSQ) ${ }^{16}$ Sleep Disturbance Severity Scale (SDSS) and a sleep diary. Participants completed the sleep diary daily throughout the trial recording sleep behaviour along with exercise performed before sleep, medication, caffeine and alcohol intake. Demographic information was also collected.Increased questionnaire scores represent a higher frequency or worsening of the mood or sleep symptom. Psychological questionnaires assessed mood (Hospital Anxiety And Depression Scale (HADS); ${ }^{17}$ Profile of Mood States (POMS)); ${ }^{18}$ and generic, health-related quality of life (HRQoL) from the Assessment of Quality of Life Questionnaire (AQoL). ${ }^{19}$

\section{Analysis}

Values are reported as means and s.d. For the sleep diary variables, means were calculated for each participant and week. The weekly values were averaged for each participant and phase of the study and group means generated from these. Missing values from the questionnaires were generated according to protocol where possible. Treatment differences were examined using univariate analyses of variance, blocking on patient and adjusting for period. The hourly aMT6s levels of the sleep laboratory participant were inspected visually Participants were asked after the final session to predict the treatment arm in which they had melatonin. All statistics were performed using SPSS v17 software (SPSS, Chicago, IL, USA). $P$-values $<0.05$ were considered statistically significant. Analyses were not able to be performed on core body temperature data as only two participants consented.

\section{RESULTS}

\section{Participant characteristics}

Eight participants aged 28-69 years (49.5 years (16)) with a mediumto-large body mass index of $18-49.2 \mathrm{~kg} \mathrm{~m}^{-2}\left(26 \mathrm{~kg} \mathrm{~m}^{-2}(10.5)\right)$ and postinjury time of 6-25 years (16.9 years (7.1)) were recruited (Table 1). One participant completed the study within the sleep laboratory and six requested home-based testing sessions. The average usual sleep onset time reported by the sample was $23: 22 \mathrm{~h}(2.1 \mathrm{~min})$. No adverse events to melatonin were reported; however, three participants had an unrelated illness during the washout period, extending it by $1-3$ weeks.

\section{Melatonin levels}

Plasma concentrations were significantly higher $(P=0.01)$ following melatonin (pre-melatonin: $17.34 \mathrm{pM} \mathrm{(14.19);} \mathrm{melatonin:}$ $43554.57 \mathrm{pM}$ (33527.11)) compared with placebo (pre-placebo: 19.26 pM (15.31); placebo: 152.06 pM (190.55))(Figure 1a). aMT6s levels were also significantly higher $(P<0.01)$ following melatonin (pre-melatonin: $0.53 \mu \mathrm{g} \mathrm{h}^{-1}$ (0.19); melatonin: $152.94 \mu \mathrm{g} \mathrm{h}^{-1}(74.51)$ ) compared with placebo (pre-placebo: $0.42 \mu \mathrm{gh}^{-1}$ (0.14); placebo: $\left.0.86 \mu \mathrm{gh}^{-1}(0.40)\right)$ (Figure 1b). The hourly aMT6s levels observed for

Table 1 Baseline participant characteristics

\begin{tabular}{|c|c|c|c|c|c|c|c|c|c|}
\hline $\begin{array}{l}\text { Participant } \\
\text { code }\end{array}$ & Sex & Age & $B M I\left(\mathrm{kgm}^{-2}\right)$ & $\begin{array}{l}\text { Injury level } \\
\text { and AIS score }\end{array}$ & $\begin{array}{c}\text { Neck circumference } \\
\text { (cm) }\end{array}$ & Abdo girth $(\mathrm{cm})$ & Years with SCl & Medications & Treatment \\
\hline $1^{a}$ & $M$ & 68 & 33.0 & $\mathrm{C} 4 \mathrm{~A}$ & 48 & 120 & 24 & $\#$ & CPAP \\
\hline $2^{b}$ & $M$ & 47 & 20.3 & $\mathrm{C} 5 \mathrm{~A}$ & 42.5 & 99.5 & 12 & + & - \\
\hline 3 & $M$ & 63 & 22.2 & C7A & 47.5 & 102 & 13 & & - \\
\hline 4 & $\mathrm{~F}$ & 30 & 18.0 & $\mathrm{C} 5 \mathrm{~A}$ & 34 & 85 & 6 & $\#+$ & - \\
\hline 5 & $\mathrm{~F}$ & 43 & 22.9 & $\mathrm{C} 5 \mathrm{~A}$ & 38 & 106 & 24 & + & - \\
\hline 6 & $M$ & 48 & 23.8 & $\mathrm{C} 4 \mathrm{~A}$ & 47.5 & 105 & 25 & & - \\
\hline 7 & $\mathrm{~F}$ & 69 & 49.2 & $\mathrm{C} 6 \mathrm{~A}$ & 51 & 183 & 19 & $\#+$ & BiPAP \\
\hline 8 & $M$ & 28 & 18.5 & $\mathrm{C} 6 \mathrm{~A}$ & 39 & 76 & 12 & \# & - \\
\hline
\end{tabular}

Abbreviations: Abdo, abdominal; AIS, American spinal injury association impairment scale; BiPap, bilevel positive airway pressure; BMI, body mass index; CPAP, continuous positive airway pressure; $\mathrm{SCl}$, spinal cord injury.

aparticipant tested in sleep laboratory.

bParticipant withdrew during the first drug period (placebo) due to unrelated illness. Medications at 1st sleep study: includes one or more of \# muscle relaxant (Baclofen), + benzodiazepines

(Diazepam, Xanax). Medications were unchanged across the testing sessions for each participant with the exception of participant 5 who was no longer taking Diazepam for the last testing session (drug period $2=$ placebo) 
a

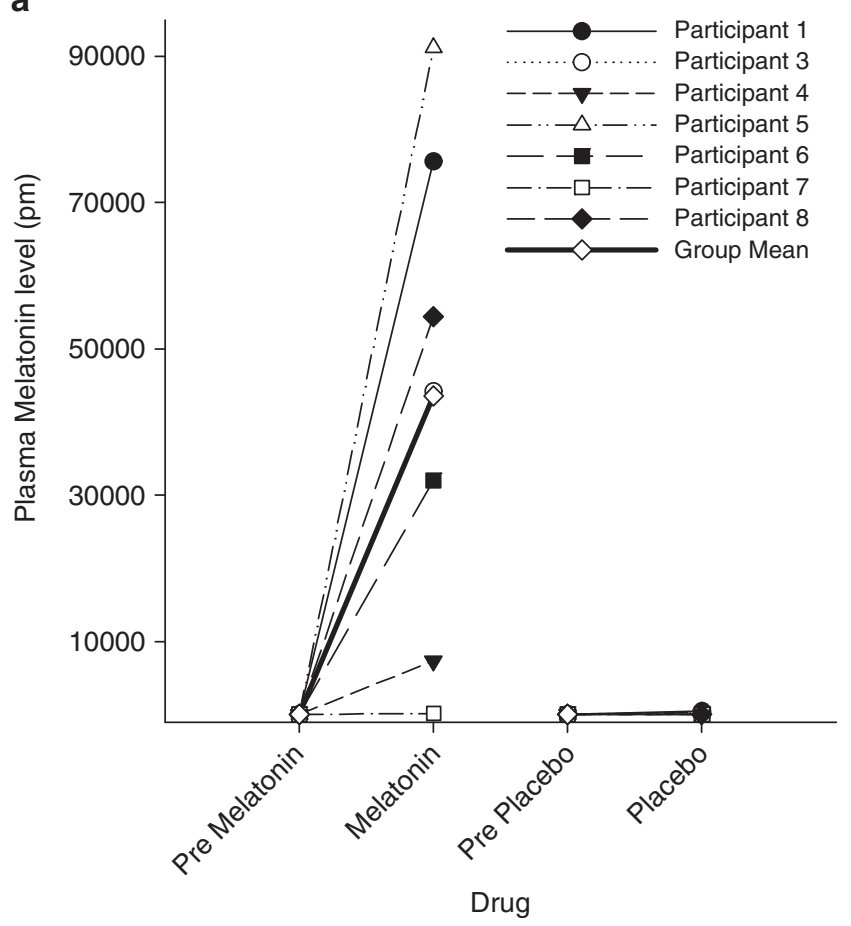

b

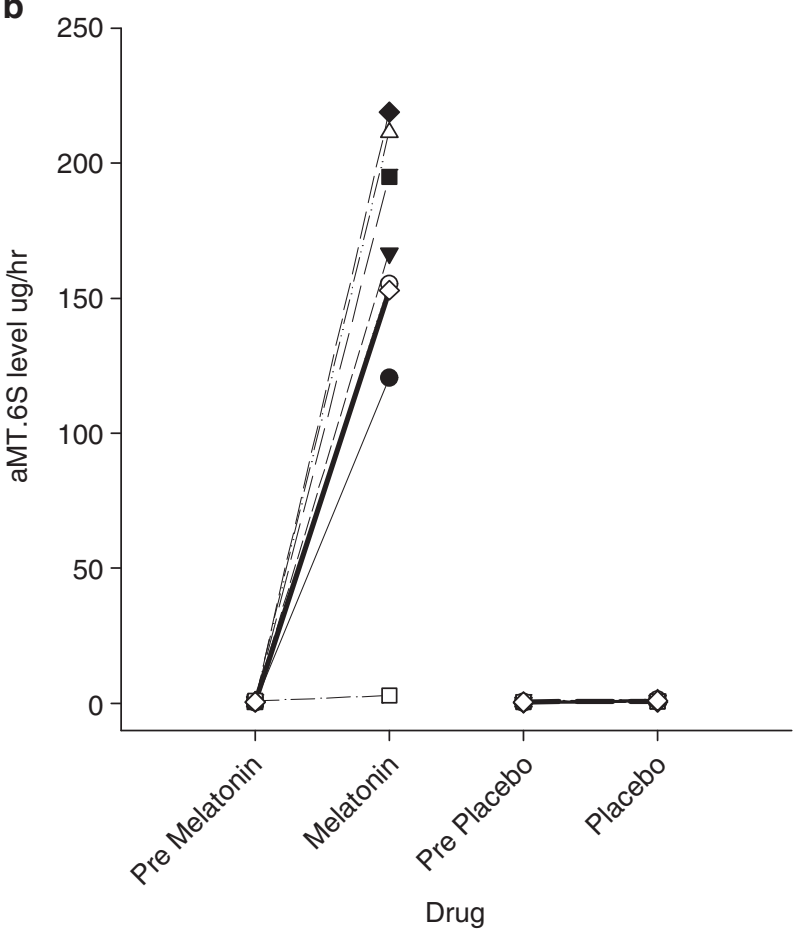

Figure 1 Individual participant (a) plasma melatonin concentrations (pM) collected approximately $1 \mathrm{~h}$ before usual sleep time and (b) aMT6s concentrations $\left(\mu \mathrm{gh}^{-1}\right)$ from urine collected during the pre-treatment and treatment phases. Note: average aMT6S levels for participant 1 (sleep laboratory participant) were generated from urine samples collected hourly between 22:00 and 07:00 hours in order to be similar to the home-based study participants who had urine collected $2 \mathrm{~h}$ before drug intake.

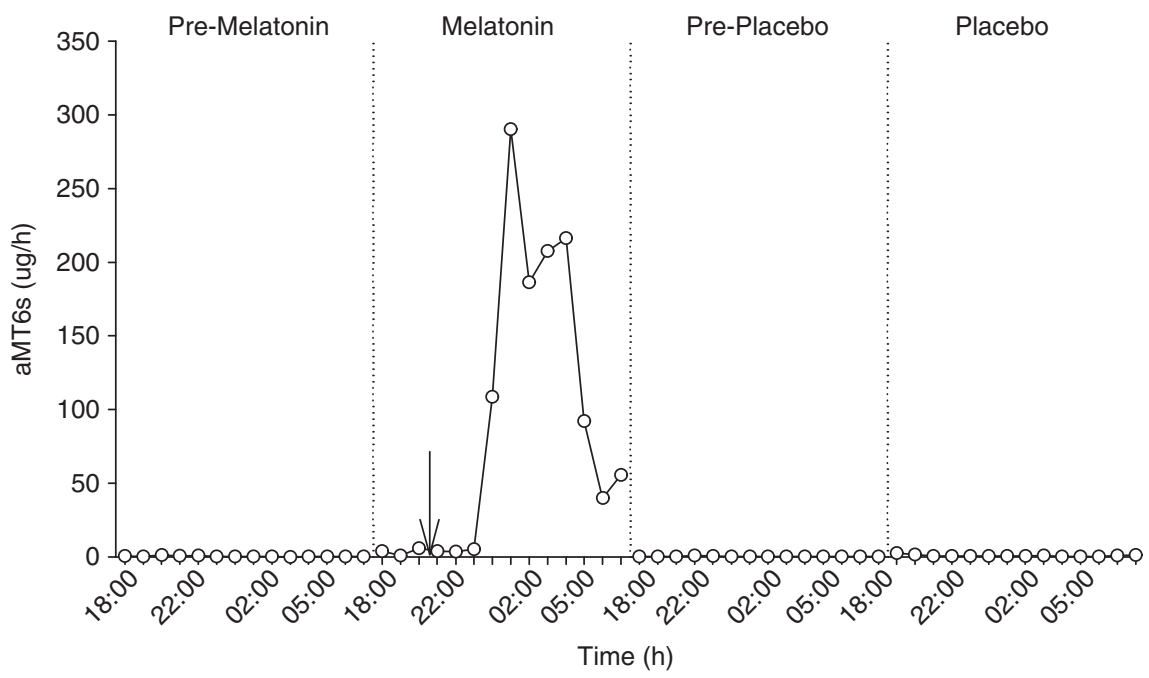

Figure 2 Hourly aMT6s concentrations $\left(\mu \mathrm{g}^{-1}\right.$ ) of the sleep laboratory participant (participant 1) from urine collected during the pre-treatment and treatment phases. Arrow indicates time of melatonin supplementation.

the sleep laboratory participant are illustrated in Figure 2. Four participants correctly identified the melatonin study arm.

\section{Sleep diary}

No significant treatment differences were observed across the sleep diary variables (Table 2). The average lights out time occurred after 23:00 h with sleep onset approximately $20 \mathrm{~min}$ later. An average of sixhour sleep was reported with one to two awakenings during the night.
The average time awake during the night was $28 \mathrm{~min}$. Morning wake time was typically $06: 40 \mathrm{~h}$ with four participants attempting to sleep again after waking (two during the placebo and two during both the treatment phases) with all but one returning to sleep (one was unsuccessful across both the treatments). With melatonin, participants fell asleep faster and were awake for a shorter period following both nocturnal awakenings and when attempting to sleep again after their morning awakening. 
Table 2 Sleep diary findings and differences between melatonin and placebo drug phases

\begin{tabular}{|c|c|c|c|c|c|}
\hline & Pre-treatment & Treatment & $\begin{array}{l}\text { Effect } \\
\text { size }(\beta)\end{array}$ & $\begin{array}{c}\text { 95\% confidence } \\
\text { interval }\end{array}$ & P-value \\
\hline \multicolumn{6}{|l|}{ LO time } \\
\hline $\begin{array}{l}\text { Melatonin } \\
\text { Placebo }\end{array}$ & $\begin{array}{c}23: 21 \text { (1 h) } \\
23: 17(43 \mathrm{~min})\end{array}$ & $\begin{array}{l}23: 10(26 \mathrm{~min}) \\
23: 14(29 \mathrm{~min})\end{array}$ & $-7 \min$ & $-21 \mathrm{~min}, 8 \mathrm{~min}$ & 0.30 \\
\hline \multicolumn{6}{|c|}{ Sleep initiation time from $L O(\mathrm{~min})$} \\
\hline $\begin{array}{l}\text { Melatonin } \\
\text { Placebo }\end{array}$ & $\begin{array}{l}25.54(28.72) \\
22.58(24.79)\end{array}$ & $\begin{array}{l}20.46(29.44) \\
24.99(25.12)\end{array}$ & -5.47 & $-19.16,8.22$ & 0.35 \\
\hline \multicolumn{6}{|l|}{ Hours sleep } \\
\hline $\begin{array}{l}\text { Melatonin } \\
\text { Placebo }\end{array}$ & $\begin{array}{l}6.02(0.81) \\
6.29(0.66)\end{array}$ & $\begin{array}{l}6.38(0.62) \\
6.11(0.69)\end{array}$ & 0.28 & $-0.18,0.74$ & 0.18 \\
\hline \multicolumn{6}{|c|}{ Number of awakenings } \\
\hline $\begin{array}{l}\text { Melatonin } \\
\text { Placebo }\end{array}$ & $\begin{array}{l}1.98(1.23) \\
1.94(1.05)\end{array}$ & $\begin{array}{l}1.60(1.26) \\
1.63(1.30)\end{array}$ & -0.01 & $-0.83,0.81$ & 0.98 \\
\hline \multicolumn{6}{|c|}{ Wake time during sleep (min) } \\
\hline Melatonin & $30.78(28.03)$ & $25.21(19.39)$ & -4.57 & $-20.22,11.08$ & 0.46 \\
\hline Placebo & $32.88(22.97)$ & $32.32(28.16)$ & & & \\
\hline \multicolumn{6}{|c|}{ Morning wake time } \\
\hline Melatonin & $6: 47$ (23 min) & $6: 44(23 \mathrm{~min})$ & $4 \min$ & $-7 \mathrm{~min}, 14 \mathrm{~min}$ & 0.41 \\
\hline Placebo & $6: 44(26 \mathrm{~min})$ & $6: 40$ (20 min) & & & \\
\hline \multirow{2}{*}{\multicolumn{6}{|c|}{ Sleep initiation time after morning awakening $(\min )^{a}$}} \\
\hline Melatonin & 36.25 & 3.50 & & & \\
\hline Placebo & 22.50 & $28.89(28.20)$ & & & \\
\hline \multicolumn{6}{|c|}{ Refreshed in morning } \\
\hline Melatonin ( & 5 & 3 & & & \\
\hline Placebo $(n)$ & 4 & 3 & & & \\
\hline \multicolumn{6}{|c|}{ Alcohol before sleep $\left(h^{\mathrm{a}}\right.$} \\
\hline Melatonin & $3.67(0.94)$ & 3.00 & & & \\
\hline Placebo & 3.03 & $3.77(0.81)$ & & & \\
\hline \multicolumn{6}{|c|}{ Caffeine before sleep $(h)^{\natural}$} \\
\hline Melatonin & 1.00 & $3.00(2.83)$ & & & \\
\hline Placebo & 1.00 & 1.07 & & & \\
\hline \multicolumn{6}{|c|}{ Medication before sleep (h) } \\
\hline Melatonin & $1.80(0.84)$ & $1.59(0.63)$ & -0.09 & $-0.31,0.13$ & 0.28 \\
\hline Placebo & $1.94(0.97)$ & $1.70(0.76)$ & & & \\
\hline \multicolumn{6}{|c|}{ Exercise before sleep (h) } \\
\hline Melatonin & $3.49(2.50)$ & $2.42(1.23)$ & -0.41 & $-3.82,3.01$ & 0.37 \\
\hline Placebo & $3.36(2.05)$ & 3.66 (1.78) & & & \\
\hline
\end{tabular}

Abbreviation: LO, lights out.

Results are mean (s.d.) unless otherwise indicated. For questions that contained analogue time values, data were transformed to decimal numeric values and time values re-established from the results for clearer interpretation. For responses that included $<$ or $>$ a value, the actual value was used in analyses (for example, $>6 \mathrm{~h}, 6 \mathrm{~h}$ was entered for analysis). Effect size is the difference between melatonin and placebo treatment results from the univariate analysis of variance model.

aUnivariate analysis of variance not generated due to low sample size $(n=1$ or $n=2$ as indicated by presence or not of s.d.).

\section{Objective sleep}

The proportion of light sleep (stages 1 and 2 sleep) significantly increased following melatonin (Table 3 ), predominantly attributable to increased stage 2 sleep $(\beta=6.76,95 \%$ confidence interval $=1.50$, 12.02, $P<0.05$; pre-melatonin: $50.02 \%$ (5.64), melatonin: $52.53 \%$ (6.00); pre-placebo: $42.99 \%$ (10.38), placebo: $45.65 \%$ (8.26)). Ambient room temperature ranged between $17.9-26.7^{\circ} \mathrm{C}$.

\section{Subjective sleep}

A moderate level of sleep disturbance (SDSS) pre and post treatment was reported (scale range 1-9: pre-melatonin: 5.00 (1.53), melatonin: 4.14 (1.35); pre-placebo: 5.14 (0.69), placebo: 4.29 (1.11). Mood
Table 3 Mean (s.d.) sleep study findings and differences between melatonin and placebo drug phases

\begin{tabular}{|c|c|c|c|c|c|}
\hline & Pre-treatment & Treatment & $\begin{array}{c}\text { Effect } \\
\text { size }(\beta)\end{array}$ & $\begin{array}{c}95 \% \\
\text { confididence }\end{array}$ & P-value \\
\hline \multicolumn{6}{|l|}{$A H I$} \\
\hline Melatonin & $19.19(10.56)$ & $17.89(10.40)$ & -0.58 & $-2.90,1.74$ & 0.55 \\
\hline Placebo & $24.89(24.83)$ & $18.11(12.01)$ & & & \\
\hline \multicolumn{6}{|l|}{ Al } \\
\hline Melatonin & $20.16(10.40)$ & $22.06(11.44)$ & 3.04 & $-4.64,10.72$ & 0.36 \\
\hline Placebo & $33.80(31.75)$ & $18.91(11.06)$ & & & \\
\hline \multicolumn{6}{|c|}{ NREM 1 and $2 \%$} \\
\hline Melatonin & 61.75 (7.03) & $62.87(7.65)$ & 10.74 & $6.80,14.68$ & 0.001 \\
\hline Placebo & $60.61(20.90)$ & $52.12(6.63)$ & & & \\
\hline \multicolumn{6}{|c|}{ NREM3 and $4 \%$} \\
\hline Melatonin & 21.77 (9.69) & $18.15(8.24)$ & -5.74 & $-12.74,1.25$ & 0.09 \\
\hline Placebo & $21.35(11.84)$ & $23.62(7.44)$ & & & \\
\hline \multicolumn{6}{|l|}{ REM total \% } \\
\hline Melatonin & $16.48(8.25)$ & $18.98(8.38)$ & -5.00 & $-11.30,1.31$ & 0.10 \\
\hline Placebo & $18.04(10.04)$ & $24.26(5.14)$ & & & \\
\hline \multicolumn{6}{|c|}{ REM latency (min) } \\
\hline Melatonin & 94.07 (49.73) & $106.57(97.47)$ & 23.17 & $\begin{array}{c}-59.41 \\
105.74\end{array}$ & 0.50 \\
\hline Placebo & $143.36(128.09)$ & $80.57(31.89)$ & & & \\
\hline \multicolumn{6}{|l|}{ TST (min) } \\
\hline Melatonin & $336.79(62.54)$ & $351.00(67.33)$ & -42.56 & $-98.85,13.73$ & 0.11 \\
\hline Placebo & $315.86(52.55)$ & $391.79(21.36)$ & & & \\
\hline
\end{tabular}

$\begin{array}{llllll}\text { Awakenings } & & & & & \\ \text { Melatonin } & 28.57(16.80) & 27.14(9.74) & -4.63 & -18.80,9.55 & 0.44 \\ \text { Placebo } & 26.29(13.90) & 31.14(17.33) & & & \\ & & & & & \\ \text { Wake time during sleep } & & & & \\ \text { Melatonin } 74.79(41.50) & 67.36(43.02) & 24.67 & -26.41,75.74 & 0.27 \\ \text { Placebo } 106.50(55.08) & 43.29(30.06)\end{array}$

Abbreviations: AHI, apnoea/hypopnoea index; AI, arousal index; NREM, non-rapid-eyemovement sleep; REM, rapid-eye-movement sleep; TST, total sleep time.

Effect size is the difference between melatonin and placebo treatment results from the univariate analysis of variance model. AHI is the number apnoeas/hypopnoeas per hour of sleep; Al is the number arousals per hour of sleep; TST is the total number of minutes of sleep; NREM 1 and $2 \%$ is the proportion of TST spent in sleep stages 1 and 2; NREM 3 and $4 \%$ is the proportion of TST spent in sleep stages 3 and 4 ; REM total \% is the proportion of TST spent in sleep stage REM: awakenings is the number of awakenings during sleep; wake time during sleep is the minutes awake after first falling asleep.

(HADS and POMS), quality of life (HRQoL) and overall sleep symptoms (BNSQ total) did not change significantly following melatonin.

Ratings on the AQoL subscale 'psychological wellbeing' were significantly better $(\beta=-0.96,95 \%$ confidence interval $=-1.48$, $-0.44, P<0.01)$ after melatonin (pre-melatonin: 3.86 (1.95); melatonin: 3.29 (1.25)) compared with placebo (pre-placebo: 3.29 (1.50); placebo: $4.29(1.25)$ ). Similarly, values on BNSQ item 12 (which asks how many hours of sleep per night the participant was having), were significantly higher after melatonin $(\beta=0.77,95 \%$ confidence interval $=0.03,1.51, P<0.05)$ after melatonin (pre-melatonin: 6.36 (0.48); melatonin: $6.43(0.79))$ compared with placebo (pre-placebo: 6.21 (0.70); placebo: $5.64(0.75))$. 


\section{DISCUSSION}

Consistent with earlier research, endogenous melatonin in those with complete tetraplegia in the present study was low. ${ }^{7}$ Exogenous melatonin significantly increased aMT6s and plasma melatonin levels compared with placebo, with the magnitude of increase being variable within the sample and considerably higher than previously reported in able-bodied people. ${ }^{5}$ The aMT6s levels of the sleep laboratory participant post supplementation resembled the physiological melatonin production curve commonly observed in the able-bodied, with the peak melatonin metabolite concentration occurring at 01:00 h. A total of $3 \mathrm{mg}$ melatonin improved subjective sleep quantity and psychological wellbeing. Sleep diary entries also indicated faster sleep initiation following melatonin. Objectively, melatonin significantly increased the proportion of light sleep with associated but non-significant reductions in the proportions of deep and rapid-eye-movement sleep.

The literature on the effect of exogenous melatonin on sleep architecture in the able-bodied is inconsistent, likely due to wide variations in dose level, protocols and characteristics of the sample and the confounding presence of other sleep disorders. Despite these differences, the increase in proportion of stage 2 sleep that we observed was similar to that which has been reported previously, ${ }^{10}$ but the importance of this difference remains unclear as the difference amounted to approximately $8 \%$ of total sleep time only. Although the crossover design of the current experiment strengthened the ability of the study to observe a difference between the conditions with seven participants, the precision of the estimates remain relatively wide reflecting the variability associated with small samples. ${ }^{20}$ The observed differences may reflect a chance finding and/or night-to-night variability, and as such, larger samples would be required to enable definitive conclusion about sleep architecture to be made.

These results are promising, but it remains unclear which dose of melatonin is optimal for improving sleep experience in people with tetraplegia as this was not a dose response, rather a proof-of-concept, preliminary study. Zhdanova et al. ${ }^{21}$ treated older insomniacs, who typically present with low nocturnal melatonin levels, with $0.1,0.3$ and $3 \mathrm{mg}$ melatonin $30 \mathrm{~min}$ before usual bedtime for 1 week. The sleep-promoting effects of $3 \mathrm{mg}$ melatonin were not greater than the lower doses which effectively raised plasma melatonin to normal nocturnal levels. The 3-mg dose, however, increased plasma melatonin beyond the normal range which continued into the next day, a finding similar to that of the sleep laboratory participant in the present study. This suggests that a dose of $3 \mathrm{mg}$ melatonin for those with diminished nocturnal melatonin production may be excessive. This larger dose may be indicated for initially retiming sleep; however, the maintenance of sleep may be better promoted with the administration of lower ongoing doses. Despite the considerable increase in melatonin following $3 \mathrm{mg}$ melatonin, it was well tolerated and no adverse events were reported. There is very little literature around how melatonin supplementation affects tetraplegia, and as such, we can only speculate as to how a person would respond to the 'reintroduction' of melatonin following a prolonged period of absence post-tetraplegic injury.

Variability in the increase of circulating melatonin within the sample could potentially be related to other medications. Benzodiazepines have been associated with a reduction in melatonin in the able-bodied. ${ }^{22}$ The use of benzodiazapines in people with complete tetraplegia is extremely common, primarily prescribed to treat pain and spasm. However, we observed a significant increase in melatonin despite this potential confounding, and as, such it seems unlikely that the effect (if any) was large.
Improvement in subjective sleep following melatonin supplementation was specific to sleep quantity and psychological wellbeing with a trend toward improved sleep initiation. Improved subjective sleep may have been secondary to the well-described hypnotic effect of melatonin which reduces the wake-promoting signals of the suprachiasmatic nucleus. ${ }^{23}$ As core temperature falls in the evening, people typically become sleepy. Melatonin has been reported to decrease core temperature and it is possible that this effect was responsible for the improved subjective sleep reports. ${ }^{24}$ Incorporation of comprehensive temperature monitoring in future research into melatonin in tetraplegia would assist in understanding these interrelationships.

Following melatonin, participants reported sleeping significantly longer and generally felt better. These improvements were attributable to a significantly poorer response following placebo, and to maintenance in response scores with melatonin. Participants identified a difference in experience between the drug phases, more importantly an improvement while on melatonin. There may have been an expectation that taking a drug would improve sleep experience and when no improvement was apparent with placebo, perhaps participants scored it lower

Home-based studies are reflective of the participants' natural sleeping conditions; however, they limit the ability to control potential confounding factors such as food intake and ambient temperature. In this study, however, home-based studies provided an alternative for participants who could not travel to the sleep laboratory due to logistical difficulties. Insufficient consent was obtained to measure core body temperature due to complications that can accompany the use of rectal probes in this population, thus precise circadian phase data are lacking. However, in this study, habitual sleep onset and wake times were stable in association with set carer routines. These external cues maintained the sleep duration within usual social bounds and there was no suggestion that any subject was substantially phase advanced or delayed.

Nightly supplementation with $3 \mathrm{mg}$ melatonin successfully elevated circulating nocturnal melatonin levels in a system where it was otherwise absent. Melatonin improved subjective sleep with participants reporting faster sleep initiation, having longer sleep duration and improved psychological wellbeing. The results of this preliminary study appear to show that melatonin may have a role in promoting sleep in tetraplegia. Further investigation of the pharmacokinetics of melatonin metabolism in this population and the relationship between temperature control and melatonin levels are warranted.

\section{DATA ARCHIVING}

There were no data to deposit.

\section{CONFLICT OF INTEREST}

The authors declare no conflict of interest.

\section{ACKNOWLEDGEMENTS}

We acknowledge the generous support of the participants, the assistance of Nucleus Network with the collection of blood samples, Professor David Kennaway and the Adelaide Research Assay Facility at the University of Adelaide for conducting the plasma melatonin and urinary 6-sulphatoxymelatonin assays and Steven Vander Hoorn for his statistical advice. We also acknowledge the Transport Accident Commission for their funding support of this research. 
1 Biering-Sorensen F, Biering-Sorensen M. Sleep disturbances in the spinal cord injured an epidemiological questionnaire investigation, including a normal population. Spinal Cord 2001; 39: 505-513.

2 Short DJ, Stradling JR, Williams SJ. Prevalence of sleep apnoea in patients over 40 years of age with spinal cord lesions. J Neurol Neurosurg Psychiatry 1992; 55: 1032-1036.

3 Berlowitz DJ, Spong J, Gordon I, Howard ME, Brown DJ. Relationships between objective sleep indices and symptoms in a community sample of people with tetraplegia. Arch Phys Med Rehabil 2012; 93: 1246-1252.

4 Giannoccaro MP, Moghadam KK, Pizza F, Boriani S, Maraldi NM, Avoni P et al. Sleep disorders in patients with spinal cord injury. Sleep Med Rev 2013; 17: 399-409. in press.

5 Brzezinski A. Melatonin in humans. N Engl J Med 1997; 336: 186-195.

6 Zeitzer JM, Ayas NT, Shea SA, Brown R, Czeisler CA. Absence of detectable melatonin and preservation of cortisol and thyrotropin rhythms in tetraplegia. J Clin Endocrino Metab 2000; 85: 2189-2196.

7 Scheer FA, Zeitzer JM, Ayas NT, Brown R, Czeisler CA, Shea SA. Reduced sleep efficiency in cervical spinal cord injury; association with abolished night time melatonin secretion. Spinal Cord 2006; 44: 78-81.

8 Wurtman RJ, Zhdanova I. Improvement of sleep quality by melatonin. Lancet 1995 ; 346: 1491.

9 Skene DJ, Arendt J. Circadian rhythm sleep disorders in the blind and their treatment with melatonin. Sleep Med 2007; 8: 651-655.

10 Spong J, Kennedy GA, Brown DJ, Armstrong SM, Berlowitz DJ. Melatonin supplementation in patients with complete tetraplegia and poor sleep. Sleep Disord 2013; 2013(Article ID 128197), 8.

11 Cohen ME, Ditunno JF Jr, Donovan WH, Maynard FM Jr. A test of the 1992 international standards for neurological and functional classification of spinal cord injury. Spinal Cord 1998; 36: 554-560.
12 Aldhous ME, Arendt J. Radioimmunoassay for 6-sulphatoxymelatonin in urine using an iodinated tracer. Ann Clin Biochem 1988; 25(Pt 3), 298-303.

13 Voultsios A, Kennaway DJ, Dawson D. Salivary melatonin as a circadian phase marker: validation and comparison to plasma melatonin. J Biol Rhythms 1997; 12: 457-466.

14 Sleep-related breathing disorders in adults: recommendations for syndrome definition and measurement techniques in clinical research. The report of an American Academy of Sleep Medicine Task Force. Sleep 1999; 22: 667-689.

15 Rechtschaffen A, Kales A. A Manual of Standardized Terminology, Techniques and Scoring System for Sleep Stages in Human Subjects. National Institutes of Health: Washington DC, 1968.

16 Partinen M, Gislason T. Basic Nordic Sleep Questionnaire(BNSQ): a quantitated measure of subjective sleep complaints. J Sleep Res 1995; 4(Suppl 1), 150-155.

17 Zigmond AS, Snaith RP. The Hospital Anxiety and Depression Scale. Acta Psychiatr Scand 1983; 67: 361-370.

18 McNair D, Lorr D, Droppleman L. Manual for the Profile of Mood States. Educational and Industrial Testing Service: San Diego: CA, USA, 1971.

19 Hawthorne G, Richardson J, Osborne R. The Assessment of Quality of Life (AQoL) instrument: a psychometric measure of health-related quality of life. Qual Life Res 1999; 8: 209-224.

20 Harvey LA. Statistical power calculations reflect our love affair with P-values and hypothesis testing: time for a fundamental change. Spinal Cord 2014; 52: 2-2.

21 Zhdanova IV, Wurtman RJ, Regan MM, Taylor JA, Shi JP, Leclair OU. Melatonin treatment for age-related insomnia. J Clin Endocrinol Metab 2001; 86: 4727-4730.

22 Kabuto M, Namura I, Saitoh Y Nocturnal enhancement of plasma melatonin could be suppressed by benzodiazepines in humans. Endocrinol Jpn 1986; 33: 405-414.

23 Sack RL, Hughes RJ, Edgar DM, Lewy AJ. Sleep-promoting effects of melatonin: at what dose, in whom, under what conditions, and by what mechanisms? Sleep 1997; 20: 908-915.

24 Krauchi K, Cajochen C, Pache M, Flammer J, Wirz-Justice A. Thermoregulatory effects of melatonin in relation to sleepiness. Chronobiol Int 2006; 23: 475-484. 